\title{
Human-Robot Collaboration for Creative and Integrated Design and Fabrication Processes
}

\author{
Shani Sharif a, T. Russell Gentry ${ }^{b}$ and Larry M. Sweet ${ }^{c}$ \\ a,b School of Architecture, Georgia Institute of Technology, USA \\ c School of Interactive Computing, Georgia Institute of Technology, USA \\ Email: shani@gatech.edu, russell.gentry@coa.gatech.edu, lsweet7@cc.gatech.edu
}

\begin{abstract}
This paper proposes a new framework for digital design to fabrication workflow to create an effective collaboration process between human designers and industrial robots, in order to redefine the relationship between design and production. This framework aims at redefining the current one-directional workflow of design to robotic fabrication that is imposed by the current robot programming and control systems, originally developed for engineering product industries. Our proposed system employs machine perception, adaptive control, and design libraries to adapt fabrication processes in real time, in parallel with human perception, learning, and guidance, to act as a catalyst of innovation for design practices, accelerating the design and production of novel building and industrial components.
\end{abstract}

Keywords -

Robotic fabrication; Human-robot collaboration; Perception; Feedback; Adaptive control

\section{Introduction}

Over the last decade, industrial robots have been adopted by architects for fabrication and assembly, as they offer flexible and multi-purpose manipulators for a variety of additive, subtractive and deformative fabrication processes [1]. Combined with the potential of generative and parametric design and modeling, robots can fabricate complex three-dimensional geometries for building components and assemblies beyond the capabilities of conventional fabrication techniques. By using industrial robots in design to fabrication processes, architects can eliminate the need for costly fixtures and setup times that hinder the cost-effectiveness of automation, especially for physical prototypes in small lot sizes. Consequently, robotic fabrication, which can be enhanced by real-time process feedback, has created an opportunity to reconsider the entire design-to-production chain. In the current design to fabrication processes, many steps in the design process are speculative, dependent on estimation and prediction of the material, tool and design behavior in the production process. By parameterization of the design aspects, and measurability of the material/tool conditions with the aid of real-time sensors feedback has created an option to push the knowledge beyond speculation and into the realm of accurate and predictable architectural production.

However, the robot control and motion programming tools that are currently adopted by designers were all originally developed for engineering product industries [2]. These technologies have been developed for industrial mass production of components with known problems and processes, and predictable outcomes. The current industrial robot control system for fabrication requires that the designer have a comprehensive view of the design object and embed detailed design and machining data in the digital model before the start of the fabrication phase (i.e. a complete specification). As a result, the process of design to fabrication is mostly a one-directional workflow, in which the designer/maker has to predict material state, tool selection, fixture positioning and robot motion planning based on prior experience. Therefore, there is a high cost and time penalty for re-work (Figure 1). In the conventional workflow, while the design conception affects the process of part production, no feedback during the action of making affects the organization and content of design model and designer's intent. There is no formal mechanism for knowledge capture and reuse.

Recent research in the field of computational design calls for the new possibilities and potentials for human and machine interactions as complementing collaborators in design-making processes. These studies intend to unify design and fabrication processes and close the divide between the act of designing and making. As Gramazio and Kohler [3] argue: "Achieving a sophisticated building component ... can be compared to methods used by manufacturers from pre-industrialized ages. Despite the similarities, today the action of material handling is indirect through the use of $\mathrm{CNC}$ machines as opposed to the instant feedback about the work in 
progress the skilled manufacturer received through the tool in his hand. With CAM, the tool is controlled through explicit routing data, which leaves no room for interpretation and adaptation." Highlighting this gap, some studies have proposed the development of new interfaces for integration of physical and digital environments. These project-specific solutions augment actuators with sensors to incorporate the real time feedback on material state over the production process [4-7].

While these projects propose creative solutions for integration of sensor input and real-time feedback into the design and fabrication processes, the solutions are project-based. Focusing on this current technological gap, the goal of this research is to develop a "framework" for a comprehensive (computer and human) feedback system to be integrated into the design to fabrication workflow. This feedback system is aimed to change the current one-directional workflow to a complete closedloop and interactive system between human designer, digital design environment, robot fabricator and the final product. In this new workflow for human-industrial robot collaboration, the design can evolve to meet the affordances of the production process, and at the same time, the production method can evolve to take advantage of feedback from computer sensing and simulation, coupled with human feedback into the process. Our proposed system will employ advanced machine perception, adaptive control, and design libraries to adapt fabrication processes in real time, in parallel with human perception, learning, and guidance, to act as a catalyst of innovation for design practices, accelerating the design and production of novel building and industrial components.

\section{Role of Design Cognition in Human- Robot Interaction in Different Design Stages}

The proposed framework for human-robot collaboration should enable cognitive technologies to essentially perform as complementary operations to those of human brain cognitive capabilities. Human brain has strong cognitive capabilities in tasks such as pattern recognition, extracting and encoding relational properties such as relative distance and parallelism, shape-based object identification and segmentation, or modeling simple dynamics of the world. However human cognition cannot perform strongly in other tasks such as recalling and executing of long or arbitrary sequences of operations $[8,9]$. The main feature of human intelligence is that it can create tools and extended cognitive systems to complement its basic modes of processing and compensate for its less strong cognitive capabilities. A human-robot collaborative system is thus successful as if it can take advantage of human cognitive system strengths and compensate for its weaknesses with robotic and computer system strengths. To maximize the benefit for a successful design and fabrication system, human and robot should utilize their strengths to the fullest (Table 1).

In general, any creative design process consists of two main stages, conceptual design stage, followed by detailed design stage, leading to final design production. In the conceptual design stage, there are many factors that have to be decided by the designer, including aesthetic of design, functional adequacy, material selection and conditions, production techniques and procedures and tools, and financial and time constraints. At this stage of the design, the impact of design decisions is very high (Wang 2002). In this stage, design decisions are made based on the design intent that are matched selectively with designer's prior production experiences based on the closest correspondence with the current design situation. In addition, the act of making, the produced physical prototype, and the ongoing designer's perception of this action transform and enrich the designer's prior organization of knowledge. However, the designer's knowledge of a specific process is never adequately detailed and precise to predict all the possible situations and outcomes of the production process. In this

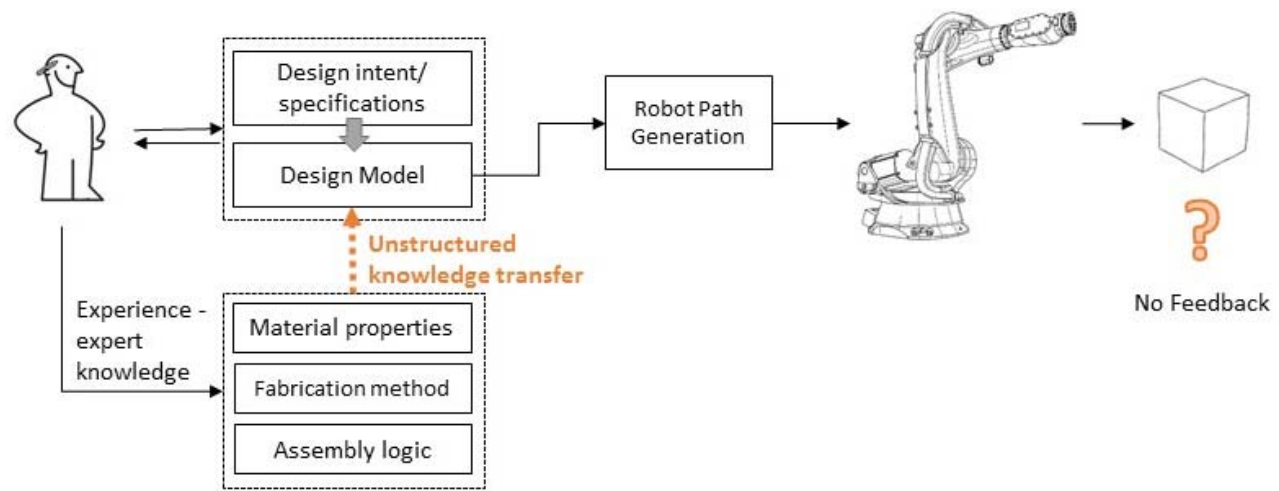

Figure 1. Current one-directional design to robotic fabrication workflow 
process, design conception and artefact production coevolve. The final design concept generated at this stage affects the product shape geometry, production material selection, as well as construction and manufacturing productivity and final product quality. In the following detailed design phase, it would be very difficult to compensate or correct the unanticipated aspects or shortcomings of the design concept developed at the conceptual design stage.

Table 1. Human Perception vs. Computer Perception, Weaknesses and Strengths [8-10]

\begin{tabular}{|c|c|}
\hline Human perception & $\begin{array}{c}\text { Robotic and Computer } \\
\text { system }\end{array}$ \\
\hline Strength & Strength \\
\hline $\begin{array}{l}\text { Creative design based on } \\
\text { design intent }\end{array}$ & $\begin{array}{l}\text { Accurate Form generation } \\
\text { with numerical inputs } \\
\text { (parametric form } \\
\text { development) }\end{array}$ \\
\hline $\begin{array}{c}\text { Alternative Form } \\
\text { development }\end{array}$ & $\begin{array}{c}\text { Speed in numerical } \\
\text { computation }\end{array}$ \\
\hline $\begin{array}{l}\text { Alternative fabrication } \\
\text { technique development }\end{array}$ & Speed in physical operation \\
\hline $\begin{array}{l}\text { Synthesizing and } \\
\text { interpolation }\end{array}$ & $\begin{array}{c}\text { Accuracy in operation } \\
\text { (computational and } \\
\text { physical), complex sequence } \\
\text { of operations }\end{array}$ \\
\hline $\begin{array}{l}\text { Detection of trends, } \\
\text { patterns, or anomalies in } \\
\text { visual data (digital and } \\
\text { physical design model) }\end{array}$ & Endurance and reliability \\
\hline $\begin{array}{l}\text { Learning from limited test } \\
\text { cases }\end{array}$ & Consistency \\
\hline \multicolumn{2}{|l|}{$\begin{array}{l}\text { Collaboration and } \\
\text { integration (people, tools, } \\
\text { senses) }\end{array}$} \\
\hline Weakness & Weakness \\
\hline $\begin{array}{l}\text { Unable to detect minor } \\
\text { deviations in the digital } \\
\text { and physical prototypes }\end{array}$ & $\begin{array}{l}\text { No creativity: difficult to } \\
\text { synthesize new rules }\end{array}$ \\
\hline $\begin{array}{l}\text { Easily tired of repetitive } \\
\text { operations }\end{array}$ & $\begin{array}{l}\text { Unable to produce alternate } \\
\text { and "out of the box" } \\
\text { solutions to compensate for } \\
\text { inadequate design solutions }\end{array}$ \\
\hline Biased and inconsistent & No common sense \\
\hline \multicolumn{2}{|l|}{ Erroneous } \\
\hline \multicolumn{2}{|c|}{$\begin{array}{l}\text { Consequently, the proposed framework should be } \\
\text { able to address different needs in design concept } \\
\text { development and detailed design development. In the } \\
\text { concept design development, the capability of the robotic } \\
\text { system in facilitating design process for designer, and } \\
\text { facilitating creativity has higher importance. In the } \\
\text { detailed design development stage, capability of the } \\
\text { system in building more accurate design prototypes, and } \\
\text { avoiding potential fabrication errors is more essential. }\end{array}$} \\
\hline
\end{tabular}

\subsection{Parametric Robot Control}

As the conventional methods of robot control and motion programming were not developed based on the needs and skills of designers, researchers in these field have focused on the development of more flexible and intuitive robot control and programming tools. These new software tools have acquired graphical programming editors such as Grasshopper. Different plug-ins such as Kuka|prc [11], HAL [12] for programming and kinematic simulation of industrial robots such as KUKA, ABB, and Universal Robots have been developed as plug-ins for Grasshopper and Dynamo. These plug-ins for graphical robot programming provide the option for architectural designers to program and simulate industrial robots directly out of the parametric modelling environment based on the geometric parameters of their designs. However, the final physical prototype outcomes are still highly dependent on the architect's predictive capability of design, fabrication, analytical and process models. While robot motion can be derived from geometric 
parameters with these tools, they provide no option for integration of material properties or fabrication intelligence into the design model to be translated into robot motions.

\subsection{Integration of design and fabrication}

While theoretically, parametric and programming tools for digital modeling of the design, would create a platform for definition of material and fabrication knowledge as generative design factors in design to fabrication models $[3,13]$, in practice, the material and fabrication factors have to be selected and modified by designers. At the current state, the human expert knowledge (based on experience and past experiments) is required to define and embed material selection, material properties, manufacturing method, assembly, and installation logic as part of the parametric CAD model, a non-automatic and error-prone process. Consequently, any mis-estimation in the anticipated design, material, and fabrication behavior and their translation into parametric rules would result in rework with high cost and time penalty. In addition, while robots have sufficient repeatability and accuracy to produce desired tool paths in theory, in practice for many applications uncertainty is introduced through variability in the process depositing or deforming the work material. Even with highest level of consideration for integration of material and fabrication logic in the parametric CAD model, unpredictable material, tool, or environmental conditions might result in deviations from anticipated results for fabricated part, as there is no instant feedback about the work in progress [14].

\section{Project Testbed}

At the current state, the proposed framework is envisioned for adoption in design research labs or commonly known as fab-labs that are environments for development and assessment of new design and production knowledge [15]. Utilizing robotic technologies at fab-labs, designers can assess and improve their design intent through fast and accurate fabrication of full-scale physical prototypes, where there is a one to one mapping between a complete CAD-CAM model, fabricated model and anticipated actual building or industrial part. Industrial robots provide new techniques for prototyping of building and industrial components out of commonly-used materials of production such as sheet metal, as opposed to model materials and techniques such as 3D printing that is still constrained by high cost, long cycle time, materials with limited strength and environmental tolerance. Prototyping with materials of production would provide the designer with process and material feedback on the actual design's weaknesses and strengths in the final production process.

As the test bed, this research focuses on sheet metal robotic manufacturing methods due to their wide range of applications in architecture and manufacturing industries. With different developing and fabrication techniques, three-dimensional forms can be made out of two-dimensional planar geometries. Robotic sheet metal fabrication is most suitable for making test models, and prototypes in small batches.

Robotic fabrication eliminates the need for time consuming and expensive methods such as drawing, punching, or hydro forming into dies. The methods for robotic fabrication with sheet metals include:

\section{Bending/folding:}

- $\quad$ Bending or straight-line folding [16, 17]

- $\quad$ Curved folding resulting in more complex threedimensional geometries [18]

Parts with complex geometries can be folded from a single planar sheet of metal without stretching, tearing or cutting, either along straight or curved lines [18-20]. Bending flat sheets along straight lines in press brakes is most common. Boxes, brackets, and similar shapes are possible by repositioning the workpiece in the brake, with subsequent bends perpendicular or oriented at other angles from prior bends. Only certain shapes are feasible, due to interference between bent shapes on the workpiece, or interference between workpiece and press brake, so human skill or advanced planning algorithms are required in design and bend sequence selection [21]. Collision detection algorithms based on segment intersection have been developed to create piecewise linear curves with a series of straight line bends, with bends all parallel to each other, with advanced planning possible for interference checking [16].

\section{Incremental sheet forming (ISF) [22, 23]}

A recent approach called "incremental forming" creates three-dimensional surfaces with continuous curvature, without constraints from straight line bending [24]. Single point and two-point incremental forming (SPIF and TPIF) tools are pressed into the metal, creating local plastic deformation. Moving the tools in discrete increments along paths digitally controlled through CNC or robots eventually creates a three-dimensional shape. Producing smooth surfaces may require very small, time consuming increments, limit material choice, and require careful characterization of material forming, lubrication, and tool shape properties, as shown by [25, 26], and [27]. Nimbalkar and Nandedkar explore the use of two sixDOF industrial robots instead of CNC machines, "Roboforming”, with the potential advantage of forming 
"undercuts" compared to CNC produced shapes, which are concave in general. However, the resultant geometries produced deviate significantly from CAD models, due to inaccuracies in models for forming process, workpiece springback, and deflections in the robots themselves due to high forces applied by the incremental forming tools.

\section{Metal driving (shrinking and stretching) [24, 28]}

Driving is one of the older manufacturing techniques for sheet metal forming by which variety of geometries can be achieved. In driving process each tool set consists of a top and a bottom tool and has sub-methods, shrinking (compressive stress), and stretching (tensile stress) which results in deformation of sheet metal. The process has been mostly manual so far, in which parts are incrementally formed by a multitude of strokes into the sheet. The robotic and numerically controlled driving which requires automated positioning and handling of sheet metal which requires complex robot tool path generation techniques ) [24, 28].

The main advantage of robotic sheet metal fabrication to other numeric controlled machines such as sheet metal CNC press brakes, shears and punching machines is the flexibility and adaptability of robotic arm for performing different fabrication and assembly methods with the appropriate end-effector. Utilizing robotic arms eliminates the need for production of dies, molds and presses. In addition, robotic arms offer fabrication methods for extremely complex geometries that otherwise could to some extend only be achieved through traditional metal craftwork in a slow and time-consuming process. The main factors in a systematic approach for robotic sheet metal fabrication includes geometric form development, analysis of material behavior during fabrication with finite element method (FEM) analysis, optimization of the fabrication process, operation sequencing, and finally robot toolpath generation.

Computer simulation of forming processes using finite element methods (FEM) are well established, so that for given shapes of forming tools (dies, molds) and material properties, the end shape can be predicted, including the effect of spring back. The more complex challenge is the inverse problem, i.e. given the desired shape, what tool geometry and material properties should be used? The first step is estimation of material properties, as shown in [29, 30]. If tool geometry is expressed parametrically, optimization methods can be developed for automated parameter identification [31], [32]. It is anticipated that based on the results of this research, the same method can be applied for other material systems and robotic fabrication methods based on the same principles.

\section{Proposed Framework for Human-Robot Interaction}

The proposed framework for human-robot interaction has two main elements (Figure 2):

1. Adaptive robot control (Based on Sensor
feedback):

- $\quad$ Matching sensor data (3D point cloud) with CAD model (in conceptual design stage)

- $\quad$ Real time sensor data + robot motion program (in detailed design-fabrication stage)

\section{Design fabrication library:}

- Design modeling (folding sequence, perforation shape, material type-thickness, fabrication method)

- Tool path library (parameterized robot tool paths, parameterized fixture points)

Addition of of an extra layer of information gathering, processing and decision making would provide a comprehensive system for human designers to work with robotic arms in the process of digital design and fabrication. Most importantly, this layer of feedbackcontrol ensures a comprehensive system for incorporation of both human and computer feedback into the design process for an effective human-robot collaboration in a bidirectional workflow from design to fabrication, and fabrication to design. The elements of this system are discussed in more detail in the text below. Note that for the successful development of this system, it is necessary to parameterize the representation of design model and the robot motion. This parametric model definition would facilitate the transformation of geometric data into fabrication data.

\subsection{Parametric Model Definition}

Developing algorithms to facilitate transformation of geometric data into fabrication data requires parameterized representation of design data. This parameterization of form would allow association of geometric information to material, robotic fabrication, and assembly attributes and properties through computational programming logics. A complete design to fabrication workflow requires association of geometric attribute to:

1. design intent (structural element, covering element, or connection part)

2. fabrication and assembly logic (folding sequence, assembly sequence, ...)

3. material properties (metal type, thickness, ductility, spring back, ...)

4. robot motion control 
5. fixture positioning and material handling (relative to robot end-effector)

6. real-time data from scanner in point cloud format

In the last few years, there have been efforts for automation of some of these aspects, such as development of tools such as KukaPRC or HAL as discussed in Section 3.2 for motion programming and control of industrial robots directly from the geometric parameters of the design, in a single modeling environment. However, the other necessary factors that are related to materiality and fabrication logic are mostly not addressed. It has to be taken into account that while some of these factors such as design intent are known to the designer in the early design stage, many of the aspects such as material type, or assembly logic are not fully defined by the designer in the conceptual design development stage. Currently, decision-making process for these factors is a non-automatic and error-prone process, based on experience and trial and error. Although these factors are not decided at conceptual design stage, creating a framework for parameterizing the attributes related to materiality and fabrication would facilitate the development of an informed design model.

\subsection{Adaptive control}

The first element in the proposed framework is the adaptive control system of robot based on real time fabrication data from a sensor system (camera, 3D scanner, or force/torque sensors. The objective of this aspect of the research is development of a system that the data from a sensor system (one or a combination of 3D scanner, camera, depth sensor, force/torque sensor...) would feedback into the design modeling or robot path generation steps of the process; create a platform for informed decision-making process. There are two main parts of the system that will use the data received from the sensor system on the state of physical prototype:

- $\quad$ Layer 1: Matching sensor data (3D point cloud) with CAD model

- $\quad$ Layer 2: Real time sensor data + robot motion program correction

Layer 1 of the adaptive control is mainly intended for conceptual design development of the process. At this layer, the 3D point cloud (or other numeric data) from the sensor will be sent back to the CAD modeling environment. By aligning the point cloud model (with parametric feature matching) with the CAD model [33], the designer can compare the deviation of the physical prototype from the intended CAD model. Based on the comparison result, designer will have the chance to editing or changing the design model based on the limitation of the fabrication method.

Layer 2 of the adaptive control is mainly intended for detailed design and fabrication stage of the process. At this layer, system uses the defined adaptive control algorithms for adjusting tool path and fixture point locations, so that the actual shape converges to the designer's intent. Based on the sensor data, the gap between the scan points and the desired shape is calculated, and the adaptive control loop adjusts the robot planning vector parameters to achieve the fabrication result closest to the design intent, defined in the computer model. These algorithms that can be defined based on the acceptable tolerance, can be really helpful for fine-tuned fabrication of each single part, so that the geometric model and robot movements are refined to minimized the tolerance and error in the final assembly of all part.

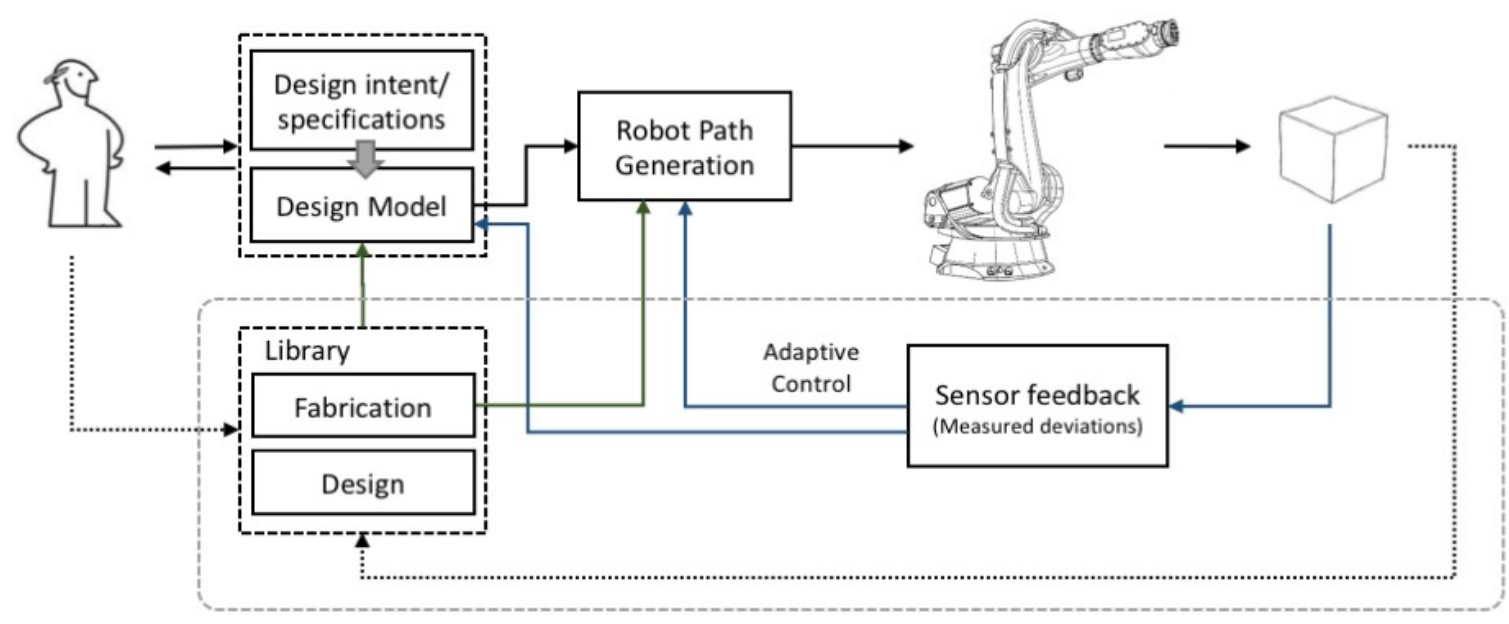

Figure 2. Proposed bi-directional design-fabrication workflow 


\subsection{Design Libraries}

Design Fabrication Library is the next element of the system that utilized the infrastructure that is layout for parametric model definition. This library has two main parts:

1. Design-fab modeling (folding sequence, perforation shape, material type-thickness, fabrication method)

2. Tool path modeling (parameterized robot tool paths, parameterized fixture points)

In the conceptual design stage, this library will assist the designers with the decision making process on the adequacy of the design choices, detailing, material selection for the selected robotic production techniques and end-effectors. The correct choices in the computermodeling environment at this step would extremely affect the success of the fabrication tests, and prevent rework and time and material waste. To achieve this goal, design library relates the mentioned parameters to sets of geometric shapes. Examples include compliant hinges for Origami-inspired sheet metal fabrications [34], and a database for optimal material and thickness identification for sheet metal folding [35].

The following example highlights one of the challenges that can solved with use of a design library. In most robotic and CNC controlled manufacturing processes, the tool path generally follows the surface of the part. This enables automatic tool path generation from CAD solid models, with adjustments made to the tool path allowing for cutting or forming tool geometry, approach vector, and process specific characteristics. Fixture points can be determined separately, their role primarily to assure stability of the workpiece. To fabricate three-dimensional sheet metal surfaces robotically, the tool path in general does not follow the surface, and location of fixture points has a strong influence on the shape. In the current metal folding process, the tool path is defined based on lots of experimentation with hand prototyping [18].

While for a given tool path, fixture locations, and material properties, the final shape can be determined through FEM simulation or experimentation; the main challenge is inverse planning problem. i.e. to go from desired end shape to tool path and fixture location [32]. Consequently, the design library will assist the designers with solutions stored in the library database. The designer selects 3D shape classes in the library that most closely matches the desired shape and material properties. If there is a close match with one of the stored classes, the stored parameterized tool path and fixture points may be used directly; if not the initial motion plan will be interpolated between adjacent classes. The designer has the option of modifying the initial tooling vector based on experience in working with the material. Using the initial tool path and fixture points, the first sample part is produced.

The design library will be developed incrementally based on both experimentation and human expert knowledge. Both real and virtual (FEM) experimentation along with statistical regression to interpolate between the results of these experiments would yield into a complete series of options for different design choices. The database will grow over time with data from continued experimentation by multiple users. For each initial and subsequent fabrication trial, scanned images for the surface produced will be added to the design library, so over time the library content will grow, filling in the gaps between adjacent designs.

\section{Conclusion}

This research addressed the main elements that are required for development of a new digital design to fabrication workflow, in order to create a structure for effective collaboration between human designers and industrial robots. We will implement these algorithms, controls and design libraries in an integrated test-bed and will test the test-bed with student designers in graduate and undergraduate level Robotic Fabrication courses at Georgia Tech.

We anticipate that by updating of design information based on fabrication sensor feedback would result in minimizing the errors through sensing and re-tooling/rework. This would also result in increasing the body of work that can be accomplished with industrial robots. In addition, by developing techniques for sheet metal fabrication, this research would create the potential for extension of this system to other materials and system. Most importantly, this workflow would construct the required infrastructure for involvement of designers in robotics design and fabrication process.

\section{References}

[1] McGee, W., M.P. de Leon, and A. Willette, Robotic Fabrication in Architecture, Art and Design 2014. Springer, 2014.

[2] Kolarevic, B., Architecture in the digital age: design and manufacturing. 2004: Taylor \& Francis.

[3] Gramazio, F., M. Kohler, and S. Oesterle, Encoding material. Architectural Design, 80(4), 2010.

[4] Mueller, S., P. Lopes, and P. Baudisch. Interactive construction: interactive fabrication of functional mechanical devices. in Proceedings of the 25th ACM. 2012.

[5] Willis, K.D., et al. Interactive fabrication: new interfaces for digital fabrication. in Proceedings of the fifth international conference on Tangible, 
embedded, and embodied interaction. ACM, 2011.

[6] Raspall, F., F. Amtsberg, and S. Peters, Material Feedback in Robotic Production, in Robotic Fabrication in Architecture, Art and Design 2014. Springer, 2014.

[7] Johns, R.L., A. Kilian, and N. Foley, Design Approaches Through Augmented Materiality and Embodied Computation, in Robotic Fabrication in Architecture, Art and Design 2014. Springer, 2014.

[8] Clark, A., Natural-born cyborgs: Minds, technologies, and the future of human intelligence. Oxford University Press, 2004.

[9] Norman, D.A., Being Analog, in The invisible computer: why good products can fail, the personal computer is so complex, and information appliances are the solution. MIT press, 1998.

[10] Halim, S., An integrated White+ Black box approach for designing and tuning stochastic local search algorithms (in Computer Science. National University of Singapore, 2009.

[11] Braumann, J. and S. Brell-Cokcan, Parametric robot control. Integrated CAD/CAM for Architectural Design, ACADIA, 2011.

[12] Schwartz, T., HAL, in Rob| Arch 2012. p. 92-101. Springer, 2012

[13] Menges, A., Material computation: Higher integration in morphogenetic design.. 82(2): p. 1421. Architectural Design, 2012.

[14] Kohler, M., F. Gramazio, and J. Willmann, The Robotic touch: How robots change architecture. Park Books, 2014.

[15] Valdes, F., A. Cavieres, and R. Gentry, A ProcessCentric Approach for Teaching Digital Fabrication, in SIGRADI. p. 400-404, Valparaiso, Chile, 2013.

[16] Liao, X. and G.G. Wang, Evolutionary path planning for robot assisted part handling in sheet metal bending. Robotics and Computer-Integrated Manufacturing. 19(5): p. 425-430. 2003

[17] Aomura, S. and A. Koguchi, Optimized bending sequences of sheet metal bending by robot. Robotics and Computer-Integrated Manufacturing, 18(1): p. 29-39. 2002.

[18] Epps, G. and S. Verma. Curved Folding: Design to fabrication process of RoboFold. in Shape Modeling International. 2013.

[19] Tachi, T. and G. Epps. Designing One-DOF mechanisms for architecture by rationalizing curved folding. in International Symposium on Algorithmic Design for Architecture and Urban Design (ALGODE-AIJ). Tokyo. 2011.

[20] Kilian, M., et al. Curved folding. in ACM Transactions on Graphics (TOG). ACM, 2008.

[21] Duflou, J.R., J. Váncza, and R. Aerens, Computer aided process planning for sheet metal bending: $A$ state of the art. Computers in industry, 56(7). 2005.
[22] Kalo, A. and M.J. Newsum, An investigation of robotic incremental sheet metal forming as a method for prototyping parametric architectural skins, in Robotic Fabrication in Architecture, Art and Design 2014. p. 33-49. Springer, 2014.

[23] Ponticel, P. Ford sheet-metal-forming technology on journey from lab to application. 2013 [cited 2016; Available from: http://articles.sae.org/12385/.

[24] Opritescu, D. and W. Volk, Automated driving for individualized sheet metal part production-A neural network approach. Robotics and ComputerIntegrated Manufacturing, 35: p. 144-150. 2015.

[25] Arshad, S., Single Point Incremental Forming. KTH Royal Institute of technology Stockholm, Sweden, 2012.

[26] Bouffioux, C., et al., Identification of material parameters to predict Single Point Incremental Forming forces. International Journal of Material Forming. 1(1): p. 1147-1150. 2008.

[27] Nimbalkar, D. and V. Nandedkar, Review of incremental forming of sheet metal components. Int J Eng Res Appl, 3(5): p. 39-51. 2013.

[28] Hoffmann, H., R. Hautmann, and R. Petry. Studies for the development of a simulation basis for numerically controlled driving of sheet metal. in Advanced Materials Research. Trans Tech, 2005.

[29] De-Carvalho, R., R. Valente, and A. AndradeCampos, Optimization strategies for non-linear material parameters identification in metal forming problems. Computers \& structures, 89(1). 2011.

[30] Chaparro, B., et al., Material parameters identification: Gradient-based, genetic and hybrid optimization algorithms. Computational Materials Science, 44(2): p. 339-346. 2008.

[31] Valente, R.A., et al., Parameter identification and shape optimization: An integrated methodology in metal forming and structural applications. Optimization and Engineering, 12(1-2). 2011.

[32] Ponthot, J.-P. and J.-P. Kleinermann, A cascade optimization methodology for automatic parameter identification and shape/process optimization in metal forming simulation. Computer Methods in Applied Mechanics and Engineering, 195(41). 2006.

[33] Ip, C.Y. and S.K. Gupta, Retrieving matching CAD models by using partial $3 D$ point clouds. ComputerAided Design and Applications, 4(5). 2007.

[34] Delimont, I.L., S.P. Magleby, and L.L. Howell, Evaluating compliant hinge geometries for origami-inspired mechanisms. Journal of Mechanisms and Robotics, 7(1). 2015.

[35] IndustrialOrigami. Industrial Origami Database. 2016 [cited 2016; Available from: http://www.industrialorigami.com/technology/prop rietary_database.cfm. 